\title{
STEM CELL THERAPY AS A POTENTIAL TREATMENT FOR GLAUCOMA
}

\author{
Kamal $M^{1}$, Amini $F^{1}$, Ramasamy $T S^{2}$ \\ 1 School of Healthy Aging, Medical Aesthetics and Regenerative Medicine, Faculty of Medicine and Health Science, UCSI \\ University, Kuala Lumpur, Malaysia. \\ 2 Department of Molecular Medicine, Faculty of Medicine, University of Malaya, Kuala Lumpur, Malaysia.
}

\section{Correspondence:}

Thamil Selvee Ramasamy

Department of Molecular Medicine, Faculty of Medicine, University of Malaya, 50603 Kuala Lumpur, Malaysia.

Email: selvee@ummc.edu.my

Tel: +603 79674719

Fax: +603 79674957

\begin{abstract}
Glaucoma is a common eye disease that can cause irreversible damage if left undiagnosed and untreated. It is one of the most common neurodegenerative diseases causing blindness. Pre-clinical studies have been carried out on animal models of glaucoma for stem cell therapy. We carried out a systematic review to determine whether stem cell therapy had the potential to treat glaucoma. Nine studies were selected based on the predetermined inclusion and exclusion criteria. Of these nine studies, eight focused on neuroprotection conferred by stem cells, and the remaining one on neuroregeneration. Results from these studies showed that there was a potential in stem cell based therapy in treating glaucoma, especially regarding neuroprotection via neurotrophic factors. The studies revealed that a brain-derived neurotrophic factor expressed by stem cells promoted the survival of retinal ganglion cells in murine glaucoma models. The transplanted cells survived without any side effects. While these studies proved that stem cells provided neuroprotection in glaucoma, improvement of vision could not be determined. Clinical studies would be required to determine whether the protection of RGC correlated with improvement in visual function. Furthermore, these murine studies could not be translated into clinical therapy due to the heterogeneity of the experimental methods and the use of different cell lines. In conclusion, the use of stem cells in the clinical therapy of glaucoma will be an important step in the future as it will transform present-day treatment with the hope of restoring sight to patients with glaucoma.
\end{abstract}

Keywords: Glaucoma, stem cell therapy, transplantation, neuroregeneration and neuroprotection, tissue degeneration, Regenerative Medicine.

\section{Introduction}

Glaucoma is an eye disease that can cause irreversible damage if left undiagnosed and untreated (1). It is one of the most common neurodegenerative diseases causing blindness which affected 60.5 million people in 2010 and is predicted to increase worldwide to 79.6 million by 2020 (2).

Glaucoma is characterised by progressive ganglion cell and optic nerve (ON) damage leading to constriction of the visual fields and later to the loss of central vision. Although it is usually associated with increased intraocular pressure (IOP), there are patients with low or normal tension glaucoma. There are also others with increased IOP, who do not experience optic nerve damage. Increased age and elevated IOP are the risk factors for developing glaucoma. Normal IOP is 10 to $21 \mathrm{mmHg}$, but it can exceed $70 \mathrm{mmHg}$ in glaucoma (3). Elevated IOP is due to increased accumulation of extracellular matrix material in the trabecular meshwork (TM) as a result of an imbalance between extracellular matrix deposition and degradation (4). Among the pathological changes that occur in glaucoma are neural tissue loss, microglia activation, tissue remodelling and disturbance of blood flow. These initial damages affect the ON axons (5). In the early stages of glaucoma, there is a progressive loss of the neural cells of the eye such as retinal ganglion cells (RGCs) and supporting cells, such as retinal pigment epithelium (RPE) (6). RGCs die in glaucoma, causing ON degeneration and disrupting the retinal connection to the brain. Neuronal death in 
glaucoma starts with a primary axonal injury, followed by the death of injured neurones and ends with secondary degeneration of neighbouring intact neurones. The neural retina and $\mathrm{ON}$ are not capable of regeneration. Therefore, at present, loss of vision in glaucoma is irreversible.

Glaucoma in many patients progresses despite treatment (7). First-line treatment includes topical selective or nonselective beta-blockers that reduce aqueous humor secretion or a topical prostaglandin analog which increases aqueous humour outflow from the eye (8). Second-line drugs are alpha-agonists and topical carbonic anhydrase inhibitors which reduce the secretion of aqueous humour. Third-line treatment options are parasympathomimetic agents such as pilocarpine, which also increases the outflow of aqueous humour from the eye. However, it causes pupil constriction resulting in blurred vision if there is central lens opacity. These drugs are not successful in all cases of glaucoma as they only delay the loss of vision, and without treating the underlying cause (1).

For patients who do not respond to anti-glaucoma medications, laser therapy may be provided. With laser trabeculoplasty, the TM is treated with argon or diode laser, resulting in a rejuvenation of the TM. This treatment is mainly used in elderly patients because the effect is short term. Other procedures include iridotomy with an $\mathrm{Nd}$ : YAG laser, iridoplasty with an argon laser and ciliary body ablation where the laser burns the aqueous producing ciliary body and is used only in advanced refractory glaucoma (8). When there is a failure in medical or laser therapy to achieve the appropriate IOP, surgery may be offered as a treatment choice. Incisional surgery involves opening drainage channels in the anterior segment of the eye to lower the IOP (9). Trabeculectomy is the most efficient glaucoma filtration procedure. However, postoperative scarring of the drainage channel causes failure of the treatment. It can be prevented by using anti-scarring drugs such as 5-fluorouracil and mitomycin-c. The surgery also poses risks such as the development of postoperative cataract and the reduction in visual acuity (8).

Evidence obtained from studies on animal models suggests that stem cells are a potential new treatment for eye diseases, including glaucoma (4). The RGC, TM and the ON head are three potential targets for stem cell therapy in glaucoma. Most of the studies have focused on replacing RGCs because their death is the final common pathway for visual loss in glaucoma (7). Pre-clinical studies are being carried out in animal models of glaucoma. However, there is currently no literature review on whether these studies have been successful.

We performed a systematic review to evaluate the results of these studies and determine the feasibility if the studies can be applied to treat glaucoma in the future. Recent advances in stem cell based therapies hold the possibility of providing replacement cells for those cells damaged by glaucoma, as well as halting the disease progression via neuroprotection. If stem cell therapy can be used to treat glaucoma, it can reduce permanent damage and blindness in patients.

\section{Methodology}

This systematic review was a qualitative research based on an extensive literature search of publications in PubMed, Google Scholar and Cochrane Library by two researchers (FA and MK) who did the search independently. The keywords used for the literature search were generated using MeSH and included glaucoma, stem cells, progenitor cells, treatment and therapeutics. Animal studies using different cells to treat glaucoma either by neuroprotective or neurodegenerative methods were included in this study. Other inclusion criteria were English language publications, all study designs, and publications from 2000 to 2016. Studies on stem cell therapy for eye conditions other than glaucoma were excluded.

The extracted data from the selected studies were entered into a data extraction table. The data included names of authors, titles of journals, titles of studies, year of publication, study designs, sample sizes, type of cell used, interventions, comparison and outcomes. A comparative evaluation of the results of each study was also made.

Because only pre-clinical studies were available, a quality assessment could not be performed. Meta-analysis could not be done due to the heterogeneity of settings, interventions and outcomes. A narrative synthesis of the included studies was thus performed.

\section{Study selection}

A total of 4309 articles were retrieved, 1874 in PubMed and 2435 in Google Scholar using the keywords individually and in combination (Figure 1). A random hand search using a combination of words was then used. The abstracts of the articles with titles that could match most of the search criteria were read through. The article was included in the review once it met all the inclusion criteria. A total of nine articles were found suitable for analysis. One study addressed the neurodegenerative potential of stem cell therapy in glaucoma; seven studies looked at the neuroprotective effects of cell therapy in glaucoma and one study investigated both neuroregenerative and neuroprotective cell therapy. The details of the characteristics and the comparison of the outcome of the studies are presented in Table 1. 


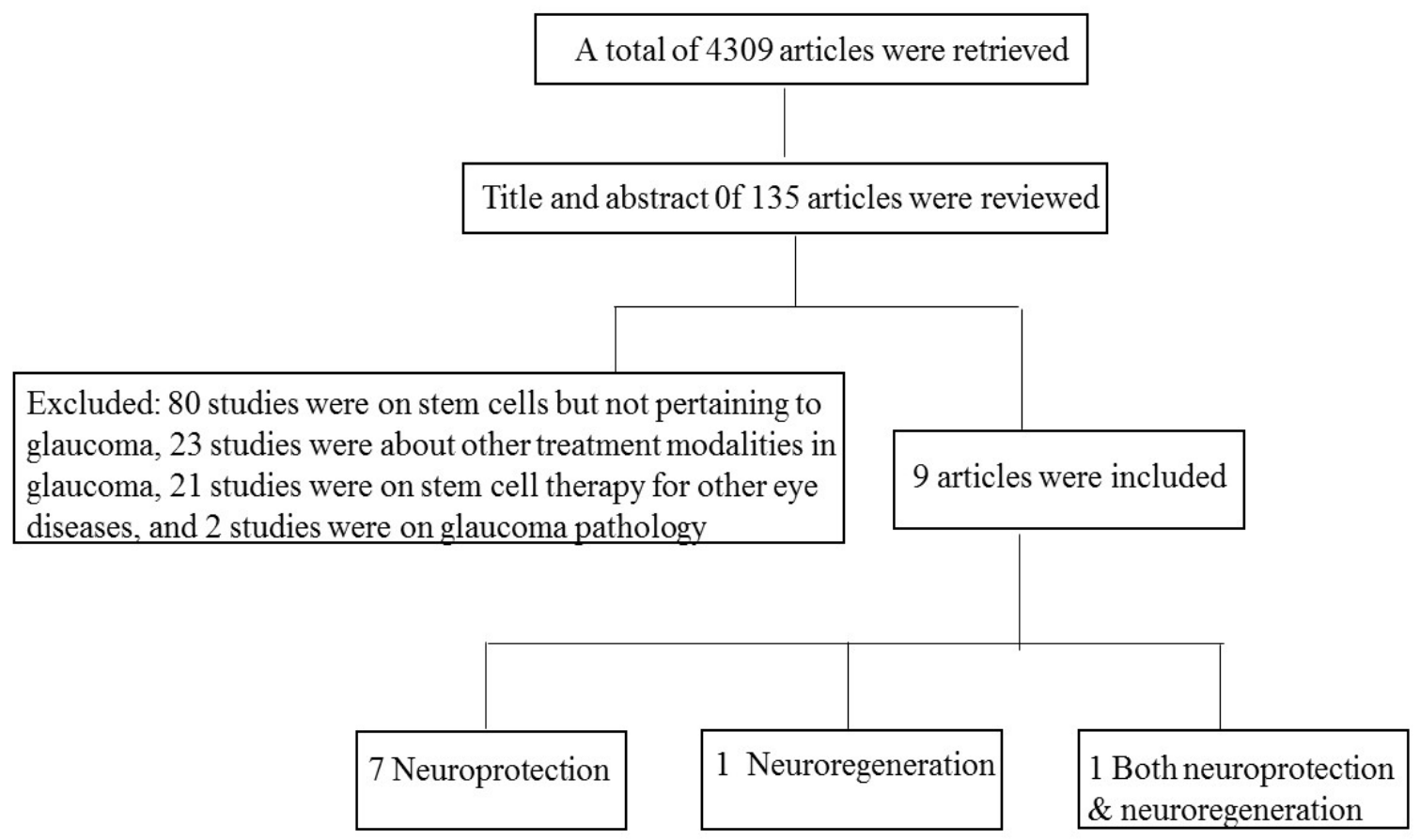

Figure 1: Flowchart of studies selection

Table 1: Selected preclinical trials of stem cells therapy in glaucoma model

\begin{tabular}{|c|c|c|c|c|c|c|c|c|}
\hline No & Year & Author & Title & $\begin{array}{l}\text { Study } \\
\text { design }\end{array}$ & Population & Intervention & Comparison & Outcome \\
\hline 1 & 2008 & $\begin{array}{l}\text { Bull et } \\
\text { al. (10) }\end{array}$ & $\begin{array}{l}\text { Human Müller } \\
\text { Stem Cell } \\
\text { (MIO-M1) } \\
\text { transplantation } \\
\text { in a murine } \\
\text { model of } \\
\text { glaucoma: } \\
\text { survival, } \\
\text { differentiation } \\
\text { and Integration. }\end{array}$ & $\begin{array}{l}\text { Animal } \\
\text { study }\end{array}$ & $\begin{array}{l}8 \text { weeks old } \\
\text { male Wistar } \\
\text { rats }\end{array}$ & $\begin{array}{l}\text { Intravitreal or } \\
\text { subretinal stem } \\
\text { cell injection } \\
\text { with concurrent } \\
\text { intraocular } \\
\text { injection of } \\
10 \mathrm{mU} / \mathrm{eye} \\
\text { chondroitinase } \\
\text { ABC, } n=17 \text { or } \\
200 \mathrm{ng} / \text { eye } \\
\text { recombinant rat } \\
\text { erythropoietin, } \\
n=12 \text {. }\end{array}$ & $\begin{array}{l}\text { Intravitreal } \\
\text { stem cell } \\
\text { injection, } \\
\text { close to RGC } \\
\text { layer, } n=46 . \\
\text { Subretinal } \\
\text { stem cell } \\
\text { injection, } \\
n=42 .\end{array}$ & $\begin{array}{l}\text { Intravitreally } \\
\text { transplanted MIO-M1 } \\
\text { cells were able to } \\
\text { survive three weeks in } \\
\text { vivo. } \\
\text { Subretinally } \\
\text { transplanted MIO-M1 } \\
\text { cells could only } \\
\text { survive for two weeks } \\
\text { after injection due to } \\
\text { invasion by immune } \\
\text { cells. } \\
\text { MIO-M1 cells that } \\
\text { were transplanted } \\
\text { either intravitreally } \\
\text { or subretinally had } \\
\text { difficulty migrating into } \\
\text { the retina. } \\
\text { MIO-M1 cells were } \\
\text { able to infiltrate the } \\
\text { retina with concurrent } \\
\text { intraocular injection of } \\
\text { either erythropoietin or } \\
\text { chondroitinase ABC. }\end{array}$ \\
\hline
\end{tabular}


Table 1: Selected preclinical trials of stem cells therapy in glaucoma model (continued)

\begin{tabular}{|c|c|c|c|c|c|c|c|c|}
\hline No & Year & Author & Title & $\begin{array}{l}\text { Study } \\
\text { design }\end{array}$ & Population & Intervention & Comparison & Outcome \\
\hline 2. & 2010 & $\begin{array}{l}\text { Johnson, } \\
\text { et al. } \\
(11)\end{array}$ & $\begin{array}{l}\text { Neuroprotective } \\
\text { Effects of } \\
\text { Intravitreal } \\
\text { Mesenchymal } \\
\text { Stem Cell } \\
\text { Transplantation } \\
\text { in Experimental } \\
\text { Glaucoma }\end{array}$ & $\begin{array}{l}\text { Animal } \\
\text { study }\end{array}$ & $\begin{array}{l}\text { 8- to } \\
\text { 12-week- } \\
\text { old male } \\
\text { Sprague- } \\
\text { Dawley } \\
\text { (SD) rats } \\
\text { and WT } \\
\text { Lewis rats }\end{array}$ & $\begin{array}{l}\text { Intravitreal MSC, } \\
\mathrm{n}=20 \text { (10 Lewis, } \\
10 \text { SD) } \\
\text { Intravenous } \\
\text { MSC, } n=9 \text { (9 SD) }\end{array}$ & $\begin{array}{l}\text { Intravitreal } \\
\text { dead MSC, } \\
n=19 \text { (10 } \\
\text { Lewis, 9 SD) } \\
\text { Intravenous } \\
\text { dead MSC, } \\
n=8 \text { (SD) } \\
\text { Intravenous } \\
\text { PBS, } n=8\end{array}$ & $\begin{array}{l}\text { Intravitreously } \\
\text { transplanted } \\
\text { mesenchymal stem } \\
\text { cells (MSC) were found } \\
\text { mainly in the vitreous } \\
\text { cavity, with a small } \\
\text { proportion of discrete } \\
\text { cells migrating into the } \\
\text { retina. } \\
\text { Intravenous } \\
\text { transplantation did not } \\
\text { result in MSC migration } \\
\text { to the retina. } \\
\text { Lewis and SD rats that } \\
\text { received intravitreal live } \\
\text { MSC grafts exhibited } \\
\text { higher RGC axon } \\
\text { survival and lower } \\
\text { rate of RGC axon loss } \\
\text { compared with those } \\
\text { that received dead } \\
\text { MSCs. } \\
\text { Intravenous injections } \\
\text { of saline, live MSCs or } \\
\text { dead MSCs, showed no } \\
\text { significant difference in } \\
\text { optic nerve damage. }\end{array}$ \\
\hline
\end{tabular}

MSC mesenchymal stem cells; SD Sprague-Dawley; RGC retinal ganglion cell

Table 1: Selected preclinical trials of stem cells therapy in glaucoma model (continued)

\begin{tabular}{|c|c|c|c|c|c|c|c|c|}
\hline No & Year & Author & Title & $\begin{array}{l}\text { Study } \\
\text { design }\end{array}$ & Population & Intervention & Comparison & Outcome \\
\hline 3. & 2006 & $\begin{array}{l}\text { Yu et al. } \\
(12)\end{array}$ & $\begin{array}{l}\text { Effects } \\
\text { of Bone } \\
\text { Marrow } \\
\text { Stromal Cell } \\
\text { Injection } \\
\text { in an } \\
\text { Experimental } \\
\text { Glaucoma } \\
\text { Model }\end{array}$ & $\begin{array}{l}\text { Animal } \\
\text { study }\end{array}$ & $\begin{array}{l}9 \text { week } \\
\text { old female } \\
\text { Wistar rats }\end{array}$ & $\begin{array}{l}\text { Intravitreal } \\
\text { injection of GFP- } \\
\text { BMSCs into the } \\
\text { glaucomatous } \\
\text { eye, } n=69\end{array}$ & $\begin{array}{l}\text { Intravitreal } \\
\text { injection of } \\
\text { PBS into the } \\
\text { glaucomatous } \\
\text { eye, } n=17\end{array}$ & $\begin{array}{l}\text { Intravitreal injection } \\
\text { of BMSC into the } \\
\text { glaucoma model eyes } \\
\text { resulted in greater RGC } \\
\text { densities compared to } \\
\text { intravitreal injection } \\
\text { of PBS. } \\
\text { The majority of GFP- } \\
\text { BMSCs within the } \\
\text { retina were found along } \\
\text { with the inner limiting } \\
\text { membrane, while only } \\
\text { a few cells integrated } \\
\text { into the nerve fibre } \\
\text { layer and ganglion cell } \\
\text { layer. } \\
\text { At both } 2 \text { and } 4 \text { weeks } \\
\text { after transplantation, } \\
\text { GFP-BMSCs that } \\
\text { expressed CNTF, GDNF, } \\
\text { BDNF, bFGF, and HGF } \alpha \\
\text { were observed in the } \\
\text { glaucomatous retinas. }\end{array}$ \\
\hline
\end{tabular}

GFP-BMSCs Green fluorescent protein-bone marrow stromal cells; RGC retinal ganglion cell; PBS Phosphate-buffered solution; CNTF ciliary neurotrophic factor; GDNF glial cell line-derived neurotrophic factor; BDNF brain-derived neurotrophic factor; bFGF basic fibroblast growth factor; HGF $\alpha$ hepatocyte growth factor $\alpha$ 
Table 1: Selected preclinical trials of stem cells therapy in glaucoma model (continued)

\begin{tabular}{|c|c|c|c|c|c|c|c|c|}
\hline No & Year & Author & Title & $\begin{array}{l}\text { Study } \\
\text { design }\end{array}$ & Population & Intervention & Comparison & Outcome \\
\hline 4. & 2009 & $\begin{array}{l}\text { Bull et } \\
\text { al. (13) }\end{array}$ & $\begin{array}{l}\text { Transplanted } \\
\text { Oligodendrocyte } \\
\text { Precursor } \\
\text { Cells Reduce } \\
\text { Neurodegeneration } \\
\text { in a Model of } \\
\text { Glaucoma }\end{array}$ & $\begin{array}{l}\text { Animal } \\
\text { study }\end{array}$ & $\begin{array}{l}8 \text { weeks old } \\
\text { male Lewis } \\
\text { rats }\end{array}$ & $\begin{array}{l}\text { Intravitreal } \\
\text { transplantation } \\
\text { at glaucoma } \\
\text { induction: } \\
3 \times 10^{4} \text { live OPCs } \\
\text { in PBS, } n=10 \\
\text { Intravitreal } \\
\text { injection before } \\
\text { glaucoma onset: } \\
3 \times 10^{4} \text { live OPCs } \\
\text { in PBS, } n=9 \text { or } \\
\text { activated } 3 \times 10^{4} \\
\text { OPCs in } 3 \mu \mathrm{LBS} \text {, } \\
\mathrm{n}=9 \text {. }\end{array}$ & $\begin{array}{l}\text { Intravitreal } \\
\text { transplantation } \\
\text { at glaucoma } \\
\text { induction: } \\
3 \mu \mathrm{L} \text { PBS, } \mathrm{n}=8 \text {, } \\
3 \times 10^{4} \text { dead OPC } \\
\text { in PBS, } \mathrm{n}=10 \\
\text { Intravitreal } \\
\text { injection before } \\
\text { glaucoma onset: } \\
3 \mu \mathrm{PBS}, \mathrm{n}=10 \text {, } \\
3 \times 10^{4} \text { dead } \\
\text { OPCs in PBS, } \\
\mathrm{n}=10 \\
\text { Intravitreal } \\
\text { injection of } \\
\text { zymosan in } 3 \mu \mathrm{L} \\
\text { PBS, n=9 }\end{array}$ & $\begin{array}{l}\text { Transplanted OPCs } \\
\text { were found to } \\
\text { survive within the } \\
\text { eye for } 4 \text { to } 12 \\
\text { weeks. } \\
\text { Activated OPCs } \\
\text { significantly } \\
\text { enhanced the } \\
\text { survival of RGCs in } \\
\text { the glaucomatous } \\
\text { eye. } \\
\text { Non-activated } \\
\text { OPCs were not } \\
\text { found to be } \\
\text { neuroprotective. } \\
\text { Transplanted OPCs } \\
\text { differentiated into } \\
\text { oligodendrocytes. } \\
\text { They had a } \\
\text { neuronal-like } \\
\text { morphology and } \\
\text { expressed bllI } \\
\text { tubulin. They also } \\
\text { differentiated } \\
\text { into astrocytes as } \\
\text { seen by astrocytic } \\
\text { morphology and } \\
\text { the presence of } \\
\text { GFAP marker }\end{array}$ \\
\hline
\end{tabular}

OPC Oligodendrocyte Precursor Cells; PBS Phosphate-buffered solution ; GFAP Glial fibrillary acidic protein

Table 1: Selected preclinical trials of stem cells therapy in glaucoma model (continue)

\begin{tabular}{|c|c|c|c|c|c|c|c|c|}
\hline No & Year & Author & Title & $\begin{array}{l}\text { Study } \\
\text { design }\end{array}$ & Population & Intervention & Comparison & Outcome \\
\hline 5. & 2011 & $\begin{array}{l}\text { Harper et } \\
\text { al. (14) }\end{array}$ & $\begin{array}{l}\text { Transplantation } \\
\text { of BDNF- } \\
\text { Secreting } \\
\text { Mesenchymal } \\
\text { Stem Cells } \\
\text { Provides } \\
\text { Neuroprotection } \\
\text { in Chronically } \\
\text { Hypertensive } \\
\text { Rat Eyes }\end{array}$ & $\begin{array}{l}\text { Animal } \\
\text { study }\end{array}$ & $\begin{array}{l}10 \text { month } \\
\text { old Brown } \\
\text { Norway } \\
\text { rats, } n=28 \\
\text { E17 rat } \\
\text { pups, } n=36\end{array}$ & $\begin{array}{l}\text { Intravitreal } \\
\text { transplantation } \\
\text { of MSC } \\
\text { engineered to } \\
\text { express BDNF } \\
\text { (BDNF-MSC) }\end{array}$ & $\begin{array}{l}\text { Intravitreal } \\
\text { transplantation } \\
\text { of MSC } \\
\text { engineered to } \\
\text { express green } \\
\text { fluorescent } \\
\text { protein (GFP-) } \\
\text { MSC }\end{array}$ & $\begin{array}{l}\text { After transplantation, } \\
\text { MSCs were mainly } \\
\text { found adjacent to } \\
\text { the GCL, integrated } \\
\text { within inner retinal } \\
\text { layers, or in the } \\
\text { vitreous cavity. } \\
\text { Eyes that received } \\
\text { BDNF-MSCs } \\
\text { displayed a greater } \\
\text { level of RGC } \\
\text { preservation than } \\
\text { eyes that received } \\
\text { GFP-MSCs. } \\
\text { BDNF-MSCs } \\
\text { preserved retinal } \\
\text { electrical activity and } \\
\text { had better afferent } \\
\text { pupillary response } \\
\text { compared to GFP- } \\
\text { MSCs. }\end{array}$ \\
\hline
\end{tabular}

BDNF-MSCs brain-derived neurotrophic factor-mesenchymal stem cells; GFP Green fluorescent protein; RGC retinal ganglion cell; GCL ganglion cell layer 
Table 1: Selected preclinical trials of stem cells therapy in glaucoma model (continued)

\begin{tabular}{|c|c|c|c|c|c|c|c|c|}
\hline No & Year & Author & Title & $\begin{array}{l}\text { Study } \\
\text { design }\end{array}$ & Population & Intervention & Comparison & Outcome \\
\hline 6. & 2012 & $\begin{array}{l}\text { Park et } \\
\text { al. (15) }\end{array}$ & $\begin{array}{l}\text { Stem Cell-Based } \\
\text { Delivery of } \\
\text { Brain-Derived } \\
\text { Neurotrophic } \\
\text { Factor Gene in } \\
\text { the Rat Retina }\end{array}$ & $\begin{array}{l}\text { Animal } \\
\text { study }\end{array}$ & $\begin{array}{l}7-8 \\
\text { weeks old } \\
\text { Sprague- } \\
\text { Dawley } \\
\text { rats }\end{array}$ & $\begin{array}{l}\text { Single unilateral } \\
\text { intravitreal } \\
\text { injection of MSC } \\
\text { with BDNF gene, } \\
n=16 \\
\text { Single unilateral } \\
\text { subretinal } \\
\text { injection of MSC } \\
\text { with BDNF gene, } \\
n=36 \text {. }\end{array}$ & $\begin{array}{l}\text { Single } \\
\text { unilateral } \\
\text { intravitreal } \\
\text { injection of } \\
\text { PBS, } n=16 \text { or } \\
\text { MSC, } n=16 \\
\text { Single } \\
\text { unilateral } \\
\text { subretinal } \\
\text { injection of } \\
\text { PBS, } n=31 \text {, or } \\
\text { MSC, } n=36\end{array}$ & $\begin{array}{l}\text { Subretinal injection } \\
\text { of MSCs produced } \\
\text { migration and } \\
\text { integration into the } \\
\text { rat retina. Some of } \\
\text { the transplanted } \\
\text { MSCs exhibited } \\
\text { morphological changes, } \\
\text { but differentiation } \\
\text { could not be } \\
\text { determined. } \\
\text { Intravitreal injection of } \\
\text { MSCs were clustered } \\
\text { in the vitreous cavity, } \\
\text { and integration into } \\
\text { the retina was not } \\
\text { detected. } \\
\text { BDNF mRNA and } \\
\text { protein levels were } \\
\text { significantly increased } \\
\text { in rats that received } \\
\text { subretinal injection } \\
\text { of transduced MSCs } \\
\text { compared with } \\
\text { those that received } \\
\text { intravitreal injection } \\
\text { of transduced MSCs, } \\
\text { non-transduced MSCs } \\
\text { and PBS. }\end{array}$ \\
\hline
\end{tabular}

MSCs mesenchymal stem cells; BDNF brain-derived neurotrophic factor

Table 1: Selected preclinical trials of stem cells therapy in glaucoma model (continued)

\begin{tabular}{|c|c|c|c|c|c|c|c|c|}
\hline No & Year & Author & Title & $\begin{array}{l}\text { Study } \\
\text { design }\end{array}$ & Population & Intervention & Comparison & Outcome \\
\hline 7. & 2002 & $\begin{array}{l}\text { Wang et } \\
\text { al. (16) }\end{array}$ & $\begin{array}{l}\text { Protection of } \\
\text { Retinal Ganglion } \\
\text { Cells Against } \\
\text { Glaucomatous } \\
\text { Neuropathy by } \\
\text { Neurotrophin- } \\
\text { Producing, } \\
\text { Genetically } \\
\text { Modified Neural } \\
\text { Progenitor Cells } \\
\text { in a Rat Model }\end{array}$ & $\begin{array}{l}\text { Animal } \\
\text { study }\end{array}$ & $\begin{array}{l}\text { Adult male } \\
\text { Sprague- } \\
\text { Dawley rats }\end{array}$ & $\begin{array}{l}\text { Intravitreal } \\
\text { transplantation } \\
\text { of genetically } \\
\text { modified neural } \\
\text { progenitors } \\
\text { producing BDNF } \\
\text { (BDNF-NPC) } \\
\text { into the left eye, } \\
\mathrm{n}=5 \\
\text { Subretinal } \\
\text { transplantation } \\
\text { of genetically } \\
\text { modified neural } \\
\text { progenitors } \\
\text { producing BDNF } \\
\text { (BDNF-NPC) } \\
\text { into the left eye, } \\
\mathrm{n}=5\end{array}$ & $\begin{array}{l}\text { Intravitreal } \\
\text { sham } \\
\text { injection, } \\
\mathrm{n}=5 \\
\text { Intravitreal } \\
\text { injection of } \\
\text { NPC into the } \\
\text { left eye, } \mathrm{n}=5 \\
\text { Subretinal } \\
\text { sham } \\
\text { injection, } \\
\mathrm{n}=5 \\
\text { Subretinal } \\
\text { injection of } \\
\text { NPC into the } \\
\text { left eye, } \mathrm{n}=5 \text {. }\end{array}$ & $\begin{array}{l}\text { Transplanted cells } \\
\text { migrated to a large } \\
\text { retinal area. } \\
\text { BDNF was expressed by } \\
\text { BDNF-NPC transplanted } \\
\text { via both intravitreal and } \\
\text { subretinal route. } \\
\text { RGC density in the } \\
\text { groups treated with } \\
\text { intravitreous or } \\
\text { subretinal injection } \\
\text { of BDNF- NPCs } \\
\text { was higher than in } \\
\text { the groups treated } \\
\text { with intravitreous } \\
\text { or subretinal sham } \\
\text { injection or NPC } \\
\text { injection. }\end{array}$ \\
\hline & & & & & & & & $\begin{array}{l}\text { RGC apoptosis was also } \\
\text { lower in groups treated } \\
\text { with BDNF-NPCs than } \\
\text { in other groups. }\end{array}$ \\
\hline
\end{tabular}

BDNF-NPC Brain-derived neural factor - neural progenitor cells; NPCs neural progenitor cells; RGC Retinal ganglion cells 
Table 1: Selected preclinical trials of stem cells therapy in glaucoma model (continued)

\begin{tabular}{|c|c|c|c|c|c|c|c|c|}
\hline No & Year & Author & Title & $\begin{array}{l}\text { Study } \\
\text { design }\end{array}$ & Population & Intervention & Comparison & Outcome \\
\hline 8. & 2013 & $\begin{array}{l}\text { Zhou et } \\
\text { al. (17) }\end{array}$ & $\begin{array}{l}\text { Neuro- } \\
\text { protection } \\
\text { of Retinal } \\
\text { Stem Cells } \\
\text { Transplantation } \\
\text { Combined with } \\
\text { Copolymer-1 } \\
\text { Immunization } \\
\text { in a Rat Model } \\
\text { of Glaucoma }\end{array}$ & $\begin{array}{l}\text { Animal } \\
\text { study }\end{array}$ & $\begin{array}{l}9 \text { weeks } \\
\text { old female } \\
\text { Sprague- } \\
\text { Dawley rats }\end{array}$ & $\begin{array}{l}\text { Immunization } \\
\text { with COP-1 } \\
\text { followed by } \\
\text { intravitreal } \\
\text { transplantation } \\
\text { of RSC (COP-1/ } \\
\text { RSCS) }\end{array}$ & $\begin{array}{l}\text { Immunization } \\
\text { with COP-1 and } \\
\text { PBS (COP-1/PBS) } \\
\text { Injection } \\
\text { of PBS and } \\
\text { transplantation } \\
\text { of RSC (PBS/ } \\
\text { RSCs) } \\
\text { Injection of } \\
\text { PBS followed } \\
\text { by another } \\
\text { injection of PBS } \\
\text { (PBS/PBS). }\end{array}$ & $\begin{array}{l}\text { Transplanted cells } \\
\text { integrated into the } \\
\text { retina. } \\
\text { The expression } \\
\text { of BDNF and } \\
\text { IGF-I in the RSCs/ } \\
\text { COP-1 group was } \\
\text { significantly higher } \\
\text { than in other groups. } \\
\text { The number of the } \\
\text { apoptotic RGCs in } \\
\text { the RSCs/COP-1 } \\
\text { group was notably } \\
\text { lower than in other } \\
\text { groups. } \\
\text { The number of RGCs } \\
\text { in the RSCs/COP-1 } \\
\text { group was higher } \\
\text { than in other groups. }\end{array}$ \\
\hline
\end{tabular}

RSC Retinal stem cells; PBS Phosphate-buffered saline; COP-1 Copolymer-1; BNDF Brain-derived neurotrophic factor; IGF-1 Insulinlike growth factor 1

Table 1: Selected preclinical trials of stem cells therapy in glaucoma model (continue)

\begin{tabular}{|c|c|c|c|c|c|c|c|c|}
\hline No & Year & Author & Title & $\begin{array}{l}\text { Study } \\
\text { design }\end{array}$ & Population & Intervention & Comparison & Outcome \\
\hline 9. & 2015 & $\begin{array}{l}\text { Emre et } \\
\text { al. (18) }\end{array}$ & $\begin{array}{l}\text { Neuroprotective } \\
\text { effects of } \\
\text { intravitreally } \\
\text { transplanted } \\
\text { adipose tissue } \\
\text { and bone } \\
\text { marrow-derived } \\
\text { mesenchymal } \\
\text { stem cells in an } \\
\text { experimental } \\
\text { ocular } \\
\text { hypertension } \\
\text { model. }\end{array}$ & $\begin{array}{l}\text { Animal } \\
\text { study }\end{array}$ & $\begin{array}{l}\text { Adult } \\
\text { female, } \\
\text { Wistar } \\
\text { albino rats }\end{array}$ & $\begin{array}{l}\text { BM-MSCs/ } \\
\text { AT-MSCs } \\
\text { group were } \\
\text { intravitreally } \\
\text { transplanted } \\
\text { to the eyes } 2 \\
\text { weeks after OHT }\end{array}$ & $\begin{array}{l}\text { BM-MSCs/ } \\
\text { AT-MSCs } \\
\text { group were } \\
\text { intravitreally } \\
\text { transplanted } \\
\text { to the eyes } 4 \\
\text { weeks after OHT }\end{array}$ & $\begin{array}{l}\text { The retinal } \\
\text { ganglion cell } \\
\text { numbers per area } \\
\text { were significantly } \\
\text { improved in stem } \\
\text { cell treated OHT } \\
\text { groups compared } \\
\text { with that in the non- } \\
\text { treated OHT group. } \\
\text { A limited number } \\
\text { of stem cells had } \\
\text { integrated into } \\
\text { the ganglion cell } \\
\text { layer and the inner } \\
\text { nuclear layer. }\end{array}$ \\
\hline
\end{tabular}

BM-MSCs bone marrow derived mesenchymal stem cells; AT-MSCs adipose tissue mesenchymal stem cells; OHT - Ocular hypertension

\section{Discussion}

The majority of studies in this review focused on neuroprotection conferred by stem cells, rather than neuroregeneration. Only the study by Bull et al. and Emre et al focused on human Müller stem cells (MIO-M1) in neuroregeneration. These cells are derived from a population of Müller glia. The MIO-M1 cells were transplanted after glaucoma induction. These cells have been shown to proliferate in vitro and have the ability to differentiate into many types of retinal neuron.

Intravitreal transplantation of employed cells was done by all 9 selected studies, but only two studies have also used subretinal transplantation and one study also used the intravenous injection of cells to evaluate any potential 
effect of the systematic application of stem cells for glaucoma. The intravitreous route was found to be more effective than the subretinal route in terms of duration of cell survival. However, cells transplanted via both routes had difficulty migrating into the retina. Erythropoeitin and chondroitinase $A B C$ were needed to increase cell migration into the retina. Erythropoietin facilitates cell integration into the retina by upregulating matrix metalloproteinase 2 expression in the central nervous system. It may also be able to enhance the migration of Müller cells in the retina. Chondroitinase $A B C$ encourages plasticity by modifying the central nervous system environment.

Johnson et al. (2010) studied whether MSC could provide neuroprotection to RGC in glaucoma. A possible contributory factor to glaucoma is the impaired RGC retrograde transport of neurotrophic factors (11). MSC was chosen because they had been shown to provide neuroprotection in degenerative central nervous system models (19). Following transplantation, they secreted neurotrophic factors and anti-inflammatory cytokines. Autologous transplantation was also possible with MSC. at study showed that intravitreal transplantation of MSC provided neuroprotection. Local transplantation had a better neuroprotective effect compared to than systemic transplantation. Intravitreally transplanted MSC survived well during the five-week study, despite no immunosuppression provided. Long-term graft survival is very promising, as it means a single treatment may be able to confer sustained neuroprotection. Intravenous transplantation of MSC proved to be unsuccessful, in contrast to other studies involving intravenous MSC transplantation in central nervous system pathologies $(20,21)$. The authors hypothesised that this might be because there is was less inflammation in glaucomatous neurodegeneration as compared to other chronic pathologies. Thus, RGC death might not produce a chemoattractive signal to draw stem cells transplanted intravenously into the retina. Two different rat breeds were used in this study because different breeds had been noted to have a dissimilar inflammatory response and protective autoimmunity (22). However, in this study, there was no difference in RGC neuroprotection by intravitreal MSC transplantation between inbred Lewis and outbred SD rats. In previous studies, intravitreal transplantation of stem cells did not affect ocular hypertension. Thus, the observed difference that glaucomatous SD rats which received live MSCs had lower IOP than glaucomatous SD rats that received dead MSCs could be due to model variability. This effect was not seen in glaucoma induced Lewis rats. Nevertheless, the transplantation of dead MSCs possibly influenced IOP elevation in SD rats, or intraocular transplantation of live MSCs reduced IOP in SD rats. Further investigation is needed to confirm whether this is indeed true.

The study by Yu et al. (2006) showed that transplantation of bone-marrow stromal cell (BMSC) did not cause inflammation or retinal structure destruction (12). However, unlike the studies by Bull et al., the transplanted cells did not express neuronal or glial markers. Thus there was no sign of differentiation. In this study, BMSC did not differentiate possibly because it was difficult to integrate into the retina. Transplanted cells faced difficulty in migrating from the vitreous cavity into the retina due to the inner limiting membrane (ILM), and without proper integration, differentiation could not happen. Thus, it is important to create a method in which transplanted cells can penetrate the ILM. Although integration occurred at a low level, RGC was protected, most probably due to neurotrophic factors and not to the replacement of cells. The trophic factor BDNF stimulates axon growth and CNTF is an RCG survival factor.

In the study by Bull et al. (2009) on oligodendrocyte precursor cells (OPC), IOP was noted to be lower in rats with glaucoma induced at eight weeks of age, as compared to rats with glaucoma induced at 16 weeks of age (13), showing that age played a role in clinical glaucoma. The OPCs had to be activated first in order to provide neuroprotection. Inflammatory cell activation of OPC is not yet fully understood but is important as it seems to trigger remyelination. Trophic factors supplied by OPCs played a part in preventing RGC death because OPCs did not migrate into neural tissue and were not directly in contact with inner retinal neurones. Compared to stem cells, progenitor cells have the capacity to differentiate but have no self-renewal capability. They are also lineagespecific precursor cells with more limited development potential. Thus, progenitor cells are more restricted than a stem cell in the type of cell it can become. In this study, OPCs were found to differentiate into astrocytes and oligodendrocytes. However, astrocytes at the optic nerve head have been seen in animal models of glaucoma progression (23). This makes transplanting OPCs, which may produce astrocytes a questionable therapy. However, in this study, the transplanted cells did not localise to the optic nerve head. Thus, the ill-effects of astrocytes can be avoided. Furthermore, astrocytes also produce trophic factors.

The study by Harper et al. (2011) showed that intravitreally transplanted MSC engineered to produce brain-derived neurotrophic factor (BDNF) were able to survive in glaucomatous eyes (14). They were mainly found adjacent to the $\mathrm{GCL}$, integrated within inner retinal layers, or in the vitreous cavity. BDNF-MSCs were found to protect the retina and optic nerve function more significantly compared to GFP-MSC when tested for pupillary light reflex and ERG. Eyes that were transplanted with BDNF-MSC also showed higher RGC preservation. However, there was no difference in optic nerve integrity between BDNF-MSCtreated eyes and GFP-MSC-treated eyes.

The study by Park et al. (2012) on MSC transduced with BDNF cDNA showed that subretinal injection had more promising results compared with intravitreal transplantation (15). These results were unlike the earlier study by Bull et al. on Müller stem cells, with more efficacious results with intravitreal transplantation, or the study by Wang et al. in which both intravitreal and subretinal transplantation 
resulted in NPC migration to the retina. However, retinal folding and retinal splitting were seen following subretinal transplantation, though there was no damage at the injection site. Even though there were morphological changes in some of the transplanted MSCs, differentiation could not be determined. This could be because the yields of MSCs that differentiated into other types of retinal cells were not enough to provide a definitive result. The method used to induce a glaucomatous condition was also different from other studies, in that optic nerve axotomy was performed instead of laser treatment to the trabecular meshwork. Axotomy mimics the condition in glaucoma because the axonal transport of neurotrophic factors is blocked, as what occurs in increased IOP. The neurones that are deprived of these factors will then die. Whether or not the different methodology influenced the results would require more comparative studies.

Wang et al. (2002) also employed a condition which mimicked glaucoma, by partially blocking ON axoplasmic flow (16). This study found that transplanted NPCs were able to migrate from the point of transplant to a large area of the retina. The genetically modified NPC producing BDNF transplanted either intravitreally or subretinally were compared with sham injection and NPC injection. BDNFNPCs were proven to be neuroprotective as there was a higher RGC density and less RGC apoptosis. There was also no sign of rejection.

Zhou et al. (2013) used RSC transplantation and vaccination with a glatiramer acetate copolymer-1 (COP-1) to study its effects on neuroprotection in a rat glaucoma model (17). In this study, many RSC were found to migrate to the retina and integrate into the nerve fibre layer and GCL. This result is better than that of the study by Harper et al., in which the transplanted MSCs were found adjacent to GCL and the study by Johnson et al., where most of the transplanted MSCs were in the vitreous cavity $(11,14)$. This was possibly due to the different types of stem cell used as well as the microenvironment suitable for cell migration. The number of the apoptotic RGCs in the RSCs/COP-1 group was also lower than in other groups, and the number of RGCs in the $\mathrm{RSC} / \mathrm{COP}-1$ group was higher than in other groups. COP-1 immunization boosts the body's protective autoimmune response (17). The increase in levels of secreted BDNF and IGF-I could be one of the mechanisms conferring neuroprotection to RGCs.

A study by Emre et al. (2015) showed that MSCs from adipose tissue and bone marrow displayed similar neuroprotective effects and that the cells injected into the vitreous cavity were integrated into the retina (18). However, the study by Yu et al. reported that bone marrow MSC did not differentiate, possibly because it was difficult to integrate into the retina (12). Transplanted cells faced difficulty in migrating from the vitreous cavity into the retina due to the inner limiting membrane (ILM). In other words, without proper integration, differentiation could not happen. Thus, it is important to create a method in which transplanted cells can penetrate the ILM. Because integration occurred at a low level, but RGC was protected, the protection was most probably due to neurotrophic factors and not to the replacement of cells. The trophic factor BDGF secreted from MSCs, stimulated axon growth and RCG survival. In all these studies, the survival rate of injected cells was varied between 2 to 12 weeks.

One of the limitations of the selected studies in their methodology was due to the method of induction of glaucoma. Glaucoma was induced by increasing ocular hypertension (IOP) in eight studies. Wang et al. used a power forcep to crush the ON close to its origin in the optic disc to induce glaucoma in the rat. It is important to bear in mind that not all types of glaucoma are due to IOP and future studies may induce glaucoma in the animal model via other mechanisms which are known to cause glaucoma. Another limitation of this review is that clinical trials were not captured; as our aim was to describe the stem cell therapy based on the rat model alone.

\section{Conclusion}

There is potential in stem cell based therapy in treating glaucoma, especially in terms of neuroprotection via secretion of neurotrophic factors. The majority of the studies showed that BDNF expressed by stem cells was able to promote the survival of RGC in rat glaucoma models.

The transplanted cells such as MSCs can survive without any side effects. This is indeed beneficial since autologous transplantation of MSC can reduce the risk of rejection as well as avoid transmission of infectious agents from donors to recipients. Ethical concerns can also be avoided by using stem cell lines derived from adults, and not embryonic or fetal tissue. Compared to therapeutic agents which require repeated administrations, stem cell transplantation provides an extended expression of protective neurotrophic factors.

Many issues need to be addressed to overcome the problems of stem cell therapy in glaucoma. The molecular pathology of glaucoma and the consequences of increased IOP at the cellular level must first be fully understood. Assessment of safety is important to ensure that the transplanted cells do not impair visual function, as the cells tended to stay within the vitreous cavity. As stem cells retain their proliferative ability, further research is needed to ensure that tumours will not be generated by the grafted cells.

The findings of these studies cannot yet be translated into clinical therapy due to the mixed results obtained from different experimental models. As the field of stem cell research and application advances and our knowledge on stem cells increases, stem cells may be used not only to rescue but also to replace retinal neurones. The use of stem cells in the clinical therapy of glaucoma will be an important step in the future as it will transform present-day treatment with the promise of restoring sight to patients with glaucoma. 


\section{Future recommendations}

Animal studies prove that stem cells can provide neuroprotection in glaucoma, but the clinical improvement of vision could not be assessed. This requires further clinical studies to determine whether the protection of RGC correlates with improvement in visual function. These limitations do not detract from the significance of the research findings, and should provide a platform for future research.

It is important that clinical trials be conducted on stem cell therapy in glaucoma. Even though the present studies utilised different types of stem cells, including retinal stem cells and neural progenitor cells, harvesting these stem cells may prove to be problematic clinically. Thus, future studies should focus more on MSC transplantation and expression of BDNF. The method of transplantation must also be refined, as pre-clinical studies have shown a discrepancy in the efficacy of intravitreal and subretinal transplantation.

Future studies should also look towards stem cells in adjuvant therapy, whereby stem cell therapy is combined with pharmacology, bio-engineering, or gene therapy. This may serve to increase the therapeutic functions of stem cells.

\section{References}

1. Lee DA and Higginbotham EJ. Glaucoma and its treatment: a review. Am J Health Syst Pharm. 2005; 62(7):691-9.

2. Quigley HA and Broman AT. The number of people with glaucoma worldwide in 2010 and 2020. Br J Ophthalmol. 2006; 90(3):262-7.

3. Khaw PT, Shah P and Elkington AR. Glaucoma--1: diagnosis. BMJ. 2004; 328(7431):97-9.

4. Bull ND and Martin KR. Concise review: toward stem cell-based therapies for retinal neurodegenerative diseases. Stem Cells. 2011; 29(8):1170-5.

5. Wierzbowska J, et al. Future possibilities in glaucoma therapy. Med Sci Monit. 2010; 16(11): Ra252-9.

6. Dahlmann-Noor A, et al. Current approaches and future prospects for stem cell rescue and regeneration of the retina and optic nerve. Can J Ophthalmol. 2010; 45(4):333-41.

7. Levin LA et al. Stem cell therapy for ocular disorders. Arch Ophthalmol. 2004; 122(4):621-7.

8. Khaw PT Shah P and Elkington AR. Glaucoma-2: Treatment. BMJ. 2004; 328(7432):156-158.

9. Chader GJ. Key needs and opportunities for treating glaucoma. Invest Ophthalmol Vis Sci. 2012; 53(5):2456-60.

10. Bull ND, Limb GA and Martin KR. Human Muller stem cell (MIO-M1) transplantation in a rat model of glaucoma: survival, differentiation, and integration. Invest Ophthalmol Vis Sci. 2008; 49(8):3449-56.

11. Johnson TV et al. Neuroprotective effects of intravitreal mesenchymal stem cell transplantation in experimental glaucoma. Invest Ophthalmol Vis Sci. 2010; 51(4):2051-9.

12. Yu S et al. Effects of bone marrow stromal cell injection in an experimental glaucoma model. Biochem Biophys Res Commun. 2006; 344(4):1071-9.

13. Bull ND, et al. Transplanted oligodendrocyte precursor cells reduce neurodegeneration in a model of glaucoma. Invest Ophthalmol Vis Sci. 2009; 50(9):4244-53.

14. Harper MM, et al. Transplantation of BDNF-secreting mesenchymal stem cells provides neuroprotection in chronically hypertensive rat eyes. Invest Ophthalmol Vis Sci. 2011; 52(7):4506-15.

15. Park HY, et al. Stem cell-based delivery of brainderived neurotrophic factor gene in the rat retina. Brain Res 2012; 1469:10-23.

16. Wang $\mathrm{N}$, et al. Protection of retinal ganglion cells against glaucomatous neuropathy by neurotrophinproducing, genetically modified neural progenitor cells in a rat model. Chin Med J (Engl). 2002; 115(9):1394-400.

17. Zhou X, Xia XB and Xiong SQ. Neuro-protection of retinal stem cells transplantation combined with copolymer-1 immunization in a rat model of glaucoma. Mol Cell Neurosci 2013; 54:1-8.

18. Emre E, et al. Neuroprotective effects of intravitreally transplanted adipose tissue and bone marrowderived mesenchymal stem cells in an experimental ocular hypertension model. Cytotherapy. 2015; 17(5):543-59.

19. Parr AM, Tator $\mathrm{CH}$ and Keating A. Bone marrowderived mesenchymal stromal cells for the repair of central nervous system injury. Bone Marrow Transplant 2007; 40(7):609-19.

20. Park HJ, et al. Mesenchymal stem cells therapy exerts neuroprotection in a progressive animal model of Parkinson's disease. J Neurochem. 2008; 107(1):14151.

21. Kim HJ, Lee JH and Kim SH. Therapeutic effects of human mesenchymal stem cells on traumatic brain injury in rats: secretion of neurotrophic factors and inhibition of apoptosis. J Neurotrauma. 2010; 27(1):131-8.

22. Cui $Q$, et al. Strain-specific differences in the effects of cyclosporin A and FK506 on the survival and regeneration of axotomized retinal ganglion cells in adult rats. Neuroscience. 2007; 146(3):986-99.

23. Hernandez MR, Miao $\mathrm{H}$ and Lukas T. Astrocytes in glaucomatous optic neuropathy. Prog Brain Res 2008; 173:353-73. 